\title{
Combining high-throughput imaging flow cytometry and deep learning for efficient species and life-cycle stage identification of phytoplankton
}

\author{
Susanne Dunker ${ }^{1,2^{*}} \mathbb{D}$, David Boho ${ }^{3}$, Jana Wäldchen ${ }^{4}$ and Patrick Mäder ${ }^{3}$
}

\begin{abstract}
Background: Phytoplankton species identification and counting is a crucial step of water quality assessment. Especially drinking water reservoirs, bathing and ballast water need to be regularly monitored for harmful species. In times of multiple environmental threats like eutrophication, climate warming and introduction of invasive species more intensive monitoring would be helpful to develop adequate measures. However, traditional methods such as microscopic counting by experts or high throughput flow cytometry based on scattering and fluorescence signals are either too time-consuming or inaccurate for species identification tasks. The combination of high qualitative microscopy with high throughput and latest development in machine learning techniques can overcome this hurdle.

Results: In this study, image based cytometry was used to collect $~ 47,000$ images for brightfield and Chl a fluorescence at $60 \times$ magnification for nine common freshwater species of nano- and micro-phytoplankton. A deep neuronal network trained on these images was applied to identify the species and the corresponding life cycle stage during the batch cultivation. The results show the high potential of this approach, where species identity and their respective life cycle stage could be predicted with a high accuracy of $97 \%$.
\end{abstract}

Conclusions: These findings could pave the way for reliable and fast phytoplankton species determination of indicator species as a crucial step in water quality assessment.

Keywords: Imaging flow cytometry, Phytoplankton, Morphology, Deep learning, CNN, Images, Image-based identification, Machine learning, High throughput cytometry, Magnification

\section{Background}

Phytoplankton monitoring is a crucial part of the biological water quality assessment $[30,47]$. Regular monitoring of indicator taxa reveals potential impairment of water life in general and specifically for human usage [11, 35, 53]. The traditional standard of phytoplankton analysis for monitoring is microscopy, being time-consuming and requiring taxonomic expertise $[5,6,11,17,18,30]$. High species diversity and detritus complicate the acquisition

\footnotetext{
*Correspondence: susanne.dunker@ufz.de

1 Department of Physiological Diversity, Helmholtz-Centre

for Environmental Research-UFZ, Permoserstraße 15, 04318 Leipzig, Germany

Full list of author information is available at the end of the article
}

of the typically required minimum of 500 cells [30] often resulting in a multi-hour counting process for the expert $[18,33]$. Due to this long analysis time, it is necessary to fix samples leading to artifacts and a loss of pigments, which could be otherwise helpful for species identification. Culverhouse et al. [13], Embleton et al. [17] and First and Drake [18] investigated the accuracy and reliability of human microscopic counting of species. First and Drake [18] asked experts with a minimum working experience greater than 7 years to identify species. The time span, selected by the authors, demonstrates how many years of expertise are thought to be necessary to guarantee reliable measurements of natural phytoplankton communities. Hofstraat et al. [28] state that taxonomy experts 
are expected to have quantification errors of $\sim 10 \%$ for dominant species and even $\sim 60 \%$ for rare species. Culverhouse [11] define four factors that limit the human performance in species identification: short-term memory of five to nine items, boredom and fatigue, recency effects and positivity bias. Furthermore, a shortage of taxonomic expertise is expected in the near future [12].

The demand for experts and the time-consuming species identification process strongly limit the feasible number of samples that can be analyzed. At the same time, there is demand for more frequent sampling, since species show high growth rates and are sensitive to environmental change, meaning that there is a high temporal and spatial variance of phytoplankton abundance $[35,48]$, especially with respect to human activities like eutrophication, climate change and introduction of invasive species. Automating this task is highly desirable, especially considering the continuous loss of experienced taxonomists with the simultaneous requirements for increased environmental monitoring.

As an alternative to traditional microscopy, analytical flow cytometers (AFC) were used for species identification $[1,5,6,19,52]$. AFC can process thousands of cells per second, making this analysis substantially faster than microscopy and enabling higher sampling frequencies [39]. AFC uses hydrodynamically focused cells in a flowing sheath stream and laser excitation to measure the corresponding scatter and several emission signals. AFC guarantees high throughput of the organisms on an individual base level. However, this technique never became widely established for species identification; instead microscopy is still common practice [18].

AFC allows a quantitative analysis of cell abundance and extraction of several individual-level parameters, like the Forward Scatter signal (indicative for cell size), the Sideward Scatter signal (indicative for granularity) and fluorescence emission values, resulting from different laser excitation, scatter and fluorescence emission channels. Scatter and fluorescence values are used to prepare two-dimensional plots, where point clouds appear [7, 33]. A high accumulation of points helps to define potential populations or sub-populations of species (gating). But the setting of gates to define a population or sub-population is subjective and strongly dependent on the expertise of the operator [7,33]. There are two possible ways from two-dimensional point clouds or principal component clouds of multidimensional data to species identification: (1) sorting of fractions with subsequent manual inspection, and (2) using pigment signatures from pure laboratory grown cultures. Many species of the same taxonomic group typically share a similar pigmentation letting their point clouds overlap. A sorted fraction of a field sample contains many different species making it impossible to uniquely assign one sorted cell to a single species one to one. Ironically, this manual inspection by sorting is similarly time-consuming as the microscopic approach. In contrast, species assignment via fluorescence pattern of pure cultures is inaccurate and extremely difficult or almost impossible to apply to field measurements, because the fluorescence emission pattern of the same species grown in the laboratory and that grown in nature could be totally different $[1,52]$. This divergence is mainly caused by differences in natural light and nutrient conditions. Phytoplankton species show a plastic response of pigmentation to be optimally acclimatized to environmental conditions [10], meaning that $\mathrm{Chl} a$ content per organic matter varies in a large range of $0.1-5 \%[21,22]$. In this study, we created variation of $\mathrm{Chl} a$ : biomass by using samples at different life cycle stages (Fig. 1).

Several authors studied machine learning techniques to improve species identification from AFC data $[1,6$, 19, 50-52]. Although the identification accuracy of these approaches is often promising, scatter properties and fluorescence emission data taken in the laboratory are not easily transferable to field samples as already mentioned. This means that calibrating identification classifier like neural networks with AFC data from laboratory samples and applying these on field samples is therefore highly erroneous [52]. This means that in practice indicator taxa can often not be uniquely identified using this approach.

Despite obvious benefits of AFC with regard to measuring speed, major drawbacks are a limited taxonomic resolution at the species level and low information contents of single scatter or fluorescence values $[8,27]$.

To overcome the mentioned limitations of the microscopic count and AFC approaches the use of imaging flow cytometry (IFC) in combination with latest computer vision techniques seems to be promising. IFC, a hybrid technology combining speed and statistical capabilities of flow cytometry with imaging features of microscopy, is rapidly advancing as a cell imaging platform that overcomes many of the limitations of current and previous techniques. Different devices are comprehensively reviewed by Dashkova et al. [14]. Using images for automated species identification has the advantage that images contain the same information that also a taxonomist would use for species identification, i.e. size, form, internal structures and conspicuous features, but are sampled much faster and being substantially richer than highly aggregated scatter or fluorescence signals, which do not contain sub-cellular fluorescence localization $[2,26]$. Furthermore, morphological properties, e.g. cell volume of species, are much less impacted by variation of environmental conditions than $\mathrm{Chl} a$ content per cell [20] and are therefore more robust for species identification. 


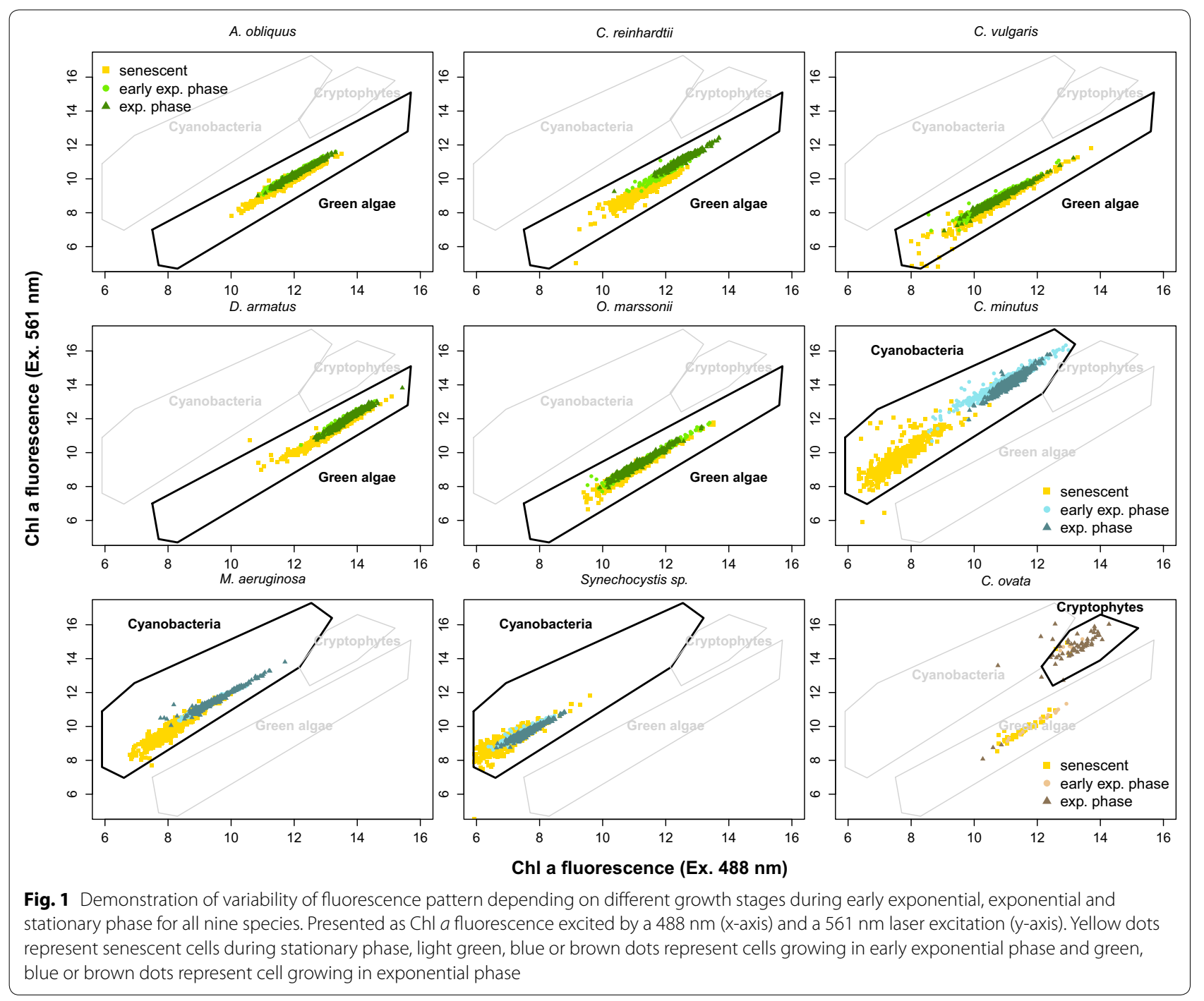

Similar to automated analysis of scatter and fluorescence signals, a number of successful approaches have been proposed for automated analysis of phytoplankton images [4, 8, 17, 23, 31, 37, 42, 45]. Many approaches to classify species from images are based on previously extracted features, such as diameter, volume or aspect ratio of the organisms. Here, the feature selection was a critical step in designing an optimal phytoplankton classification system. Deriving highly informative and complementary features is essential for high classification performance, but the process is labor-intensive, requires domain knowledge and is often subjective. Deep artificial neural networks $(\mathrm{CNN})$ automate these critical feature extraction steps by learning a suitable representation of the training data and by systematically developing a robust classification model [49]. CNNs are increasingly used in imaged based phytoplankton identification [31,
37]. However, a full automation of microscopic phytoplankton species measurement in combination with $\mathrm{CNN}$ was not shown yet. Furthermore, analyzing and predicting life stages were neglected although it reveals important additional information about the physiological state of the phytoplankton species.

The overall aim of this study is (a) to show whether species of nano- and microplankton can automatically be identified using deep neuronal networks, (b) to evaluate whether also their life cycle stage can be identified, and (c) to determine the most appropriate combination of available image channels for (a) and (b).

\section{Material and methods}

\section{Species}

Tested species [Acutodesmus obliquus (SAG 276-3a, formerly called: Scenedesmus obliquus (Turpin) Kützing, $S$. 
acutus $f$. alternans), Chlamydomonas reinhardtii (SAG 11-32b), Chlorella vulgaris (SAG 211-11b), Chroococcus minutus (SAG 41.79), Cryptomonas ovata (SAG 979-3), Desmodesmus armatus (SAG 276-4 d, formerly called: Scenedesmus quadricauda, Scenedesmus armatus Chodat), Microcystis aeruginosa (SAG 1450-1), Oocystis marssonii (SAG 257-1) and Synechocystis sp. (PCC 6803)] (overview in Table 1) were purchased from Culture Collection of Algae EPSAG (Göttingen, Germany) and Pasteur Culture Collection (Paris, France), following the Microbial Resource Research Infrastructure (MIRRI) Best Practice Manual on Access and Benefit Sharing. All strains were cultivated in different media for 144 days (Bold's Basal Medium for green algae and the cryptophyte according to Bischoff [3]: $\mathrm{NaNO}_{3}=250 \mathrm{mg} \mathrm{L} \mathrm{L}^{-1}$, $\mathrm{CaCl}_{2} 7 \mathrm{H}_{2} \mathrm{O}=25 \mathrm{mg} \mathrm{L}{ }^{-1}, \mathrm{MgSO}_{4} 7 \mathrm{H}_{2} \mathrm{O}=75 \mathrm{mg} \mathrm{L}^{-1}$, $\mathrm{KH}_{2} \mathrm{PO}_{4}=175 \mathrm{mg} \mathrm{L} \mathrm{L}^{-1}, \mathrm{~K}_{2} \mathrm{HPO}_{4} 3 \mathrm{H}_{2} \mathrm{O}=98 \mathrm{mg} \mathrm{L} \mathrm{L}^{-1}$, $\mathrm{NaCl}=25 \mathrm{mg} \mathrm{L}^{-1}$, Fe-EDTA $=1 \mathrm{~mL} \mathrm{~L}^{-1}$, micronutrient solution $=2 \mathrm{~mL} \mathrm{~L}^{-1}$ and Zehnder-Medium for cyanobacteria according to Staub [46]: $\mathrm{NaNO}_{3}=467 \mathrm{mg} \mathrm{L}^{-1}$, $\mathrm{Ca}\left(\mathrm{NO}_{3}\right){ }_{2} 4 \mathrm{H}_{2} \mathrm{O}=59 \mathrm{mg} \mathrm{L}^{-1}, \mathrm{MgSO}_{4} 7 \mathrm{H}_{2} \mathrm{O}=25 \mathrm{mg} \mathrm{L}^{-1}$,

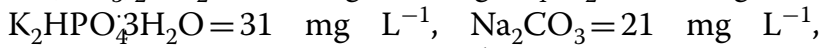
Fe-EDTA-complex $=10 \mathrm{~mL} \mathrm{~L}^{-1}$, micronutrient solution $=0.08 \mathrm{~mL} \mathrm{~L}^{-1}$ ) in a $14 / 10$ light/dark cycle on shaking tables with a light intensity of $80 \mu \mathrm{mol}$ photons $\mathrm{m}^{-2} \mathrm{~s}^{-1}$ and $20^{\circ} \mathrm{C}$ in batch culture. At different time points early exponential (day 9 after inoculation), exponential (day 23 after inoculation) and stationary phase (day 144 after inoculation). All species were selected to be (1) common freshwater species, (2) growing under meso-to eutrophic conditions (http://www.algaebase.org, [38], Table 1) and (3) similar in morphology and size range to exhaust the limits of species classification. According to Reynolds [41] and Palmer [36] some of the selected species belong to indicator genera (A. obliquus and D. armatus formerly called Scenedesmus), Chlamydomonas, Chlorella, Cryptomonas, Microcystis and Oocystis and are marked in Table 1 accordingly. In addition M. aeruginosa is a common harmful bloom forming species.

\section{Instrument settings}

Phytoplankton samples were measured with a special-order laboratory based imaging flow cytometer ImageStream ${ }^{\circledR X}$ MK II (Amnis part of EMD Millipore, Darmstadt, Germany), able to measure brightfield images and fluorescence images simultaneously. The instrument was chosen to measure nano- and microplankton species by taking images with a high sampling rate at $60 \times$ magnification and a comparably good resolution. As basis of an automated routine, many thousands of images were acquired within minutes resulting in sufficient images to train a successful deep learning classifier.

As sheath-fluid Dulbecco's phosphate buffered saline w/o calcium, w/o magnesium (Biowest, Nuaillé, France) was used. Particles of interest (excluding speed calibration beads) were collected with a $488 \mathrm{~nm}$ laser intensity of $0.1 \mathrm{~mW}$. The laser configuration is unique and specifically adapted to the measurement of phytoplankton cells allowing a first separation of different spectral groups and subsequent species identification by morphological information (patent submission PCT/EP2017/075553).

For each species $\sim 50 \mu \mathrm{L}$ of sample were used. Data acquisition was finished, when 5000 events were measured or alternatively when a time of $\sim 20$ min had elapsed.

All brightfield and Chl $a$ fluorescence images (488 nm excitation/642-745 $\mathrm{nm}$ emission) were automatically taken at $60 \times$ magnification with a numeric aperture of 0.9 , a pixel size of $0.3 \times 0.3 \mu \mathrm{m}$ and a $40 \times 170 \mu \mathrm{m}$ field of view. For CNN training, only viable (Chl $a$ containing)

Table 1 Overview about investigated species for strain identity, culture medium, cell size and weighted average tolerated Total phosphorus range from a global dataset (according to Phillips et al. [38], Supplementary material)

\begin{tabular}{|c|c|c|c|c|}
\hline & Strain & Taxonomic group & Medium & TP-range $\left(\mu \mathrm{g} \mathrm{L}^{-1}\right)^{\mathrm{a}}$ \\
\hline Acutodesmus obliquus ${ }^{\mathrm{b}, \mathrm{c}}$ & SAG 276-3a & Green algae & $\mathrm{BBM}$ & $25-90$ \\
\hline Chlamydomonas reinhardtiit, & SAG 11-32b & Green algae & BBM & $12-41$ \\
\hline Chlorella vulgaris ${ }^{\mathrm{b}, \mathrm{c}}$ & SAG 211-11b & Green algae & BBM & $27-87$ \\
\hline Chroococcus minutus & SAG 41.79 & Cyanobacteria & Z-Medium & $16-60$ \\
\hline Cryptomonas ovata & SAG 979-3 & Cryptophyte & BBM & $12-41$ \\
\hline Desmodesmus armatus ${ }^{b, c}$ & SAG 276-4d & Green algae & BBM & $25-90$ \\
\hline Microcystis aeruginosa ${ }^{c}$ & SAG 1450-1 & Cyanobacteria & Z-Medium & $38-108$ \\
\hline Oocystis marssonii & SAG 257-1 & Green algae & BBM & $8-28$ \\
\hline Synechocystis sp. & PCC 6803 & Cyanobacteria & Z-Medium & $21-62$ \\
\hline
\end{tabular}

\footnotetext{
a Tolerated range of total phosphorus (TP) according to Phillips et al. [38]

b Indicator genus according to Palmer [36]

c Indicator genus according to Reynolds [41]
} 
cells were selected based on a two-dimensional plot of cell area and Chl $a$ fluorescence intensity.

\section{Dataset}

The dataset consisted of 46,797 brightfield and 46,797 $\mathrm{Chl} a$ fluorescence images of nine species (Figs. 2 and 3). For each single cell, a brightfield and the respective $\mathrm{Chl} a$ image was used. Images were collected at three different stages of batch-culture, during early exponential, exponential and stationary phase. For almost all species, most images were available in the exponential phase (Fig. 2).

The complete dataset was split into three sets for training, validation and testing in the proportion 80:10:10. All images were center cropped to an equally sized rectangle. In order to gain a more robust and better generalizing classifier, the images in the training set were additionally augmented in the following ways: flipped horizontal, flipped vertically, adjusted in brightness $\pm 12.5 \%$, adjusted in saturation $\pm 50.0 \%$, adjusted in contrast $\pm 12.5 \%$, and adjusted in hue $\pm 20 \%$.

\section{Classifier}

As the images taken with the imaging flow cytometer have a lower image quality than a standard microscopic image, it is necessary to take advantage of a powerful deep learning model (classifier) to identify species based on imaging flow cytometric data sets [9]. Therefore, preprocessed images were finally used to train a CNN deep learning model, a common standard deep learning network for visual input. A CNN model uses several layers (input, hidden and output layer) with different image pattern information in a feed-forward mode.

We trained such a CNN classifier on the described training data. More specifically, a residual network architecture (ResNet v2) with 50 convolution layers [24], winning the prestigious ImageNet competition in 2015 and beating for the first time a human performing the same classification task, was used. Transfer learning is a common procedure for training of classifiers with less than $\sim 1 \mathrm{M}$ images [54]. That is, we used a network that was pre-trained on the large-scale ImageNet ILSVRC 2012 dataset (http://image-net.org/challenges/LSVRC /2012/) before utilized for our training. Training used a batch size of 32, with a learning rate of 0.0003 and was terminated after 80,000 steps.

In order to assess the characteristic information conveyed per captured image channel in separation and in complementation to each other, we trained four classifiers: (1) brightfield images alone, (2) Chl $a$ fluorescence images alone, (3) all brightfield-Chl $a$ fluorescence images and (4) merged brightfield-Chl $a$ fluorescence images. Classifiers (1) and (2) assess the individual species characteristics conveyed per channel. Classifier (3) is trained with a mixture of images from both channels assessing whether there is complementary information in the channels and representation per class helps in generalizing and creating a more robust classifier. Finally, classifier (4) is trained with two-channel images containing the full amount of available information at training and classification time.

Simultaneously analysis of brightfield and Chl $a$ fluorescence images, is expected as best way of classification. Brightfield images contain morphological information, while Chl $a$ fluorescence images reveal chloroplast morphology, both being important parameters for traditional taxonomic identification [29]. It is hypothesized that both images carry complementary information helpful for species identification.

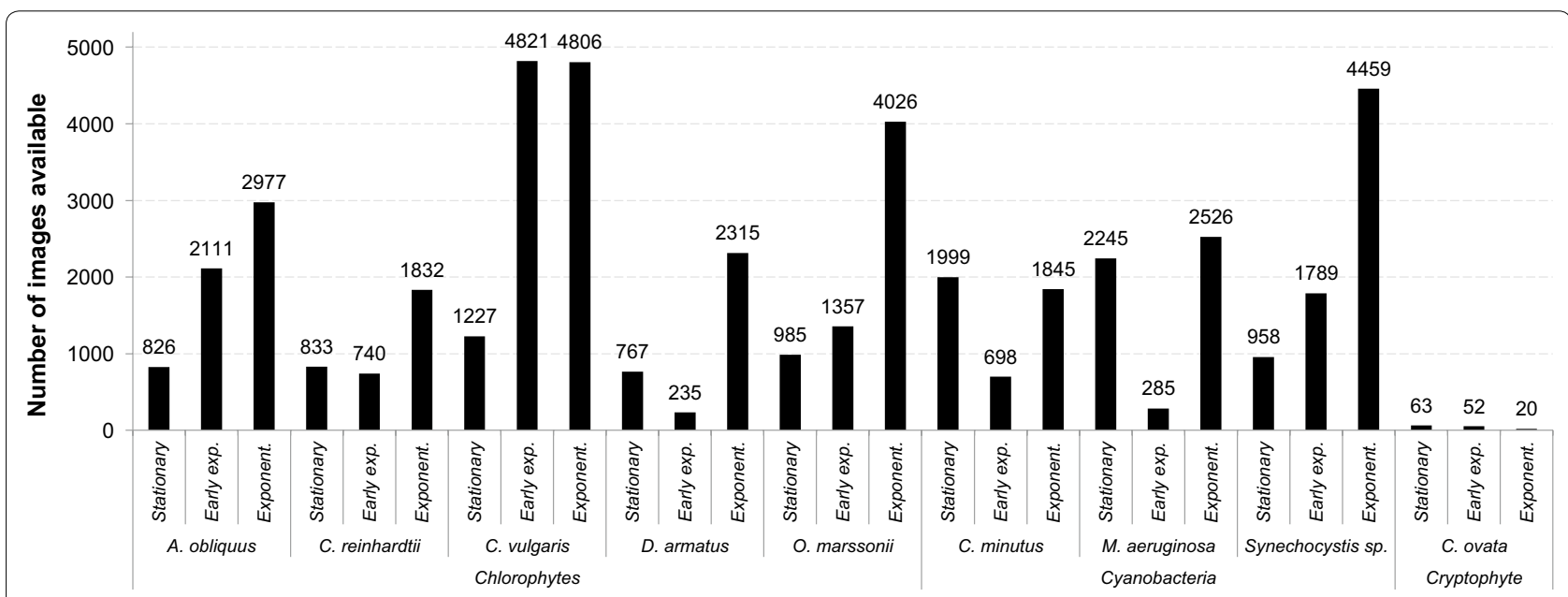

Fig. 2 Overview about number of images (brightfield or Chl a fluorescence images respectively) included per species and life cycle stage (stationary phase, early exponential phase and exponential phase) 


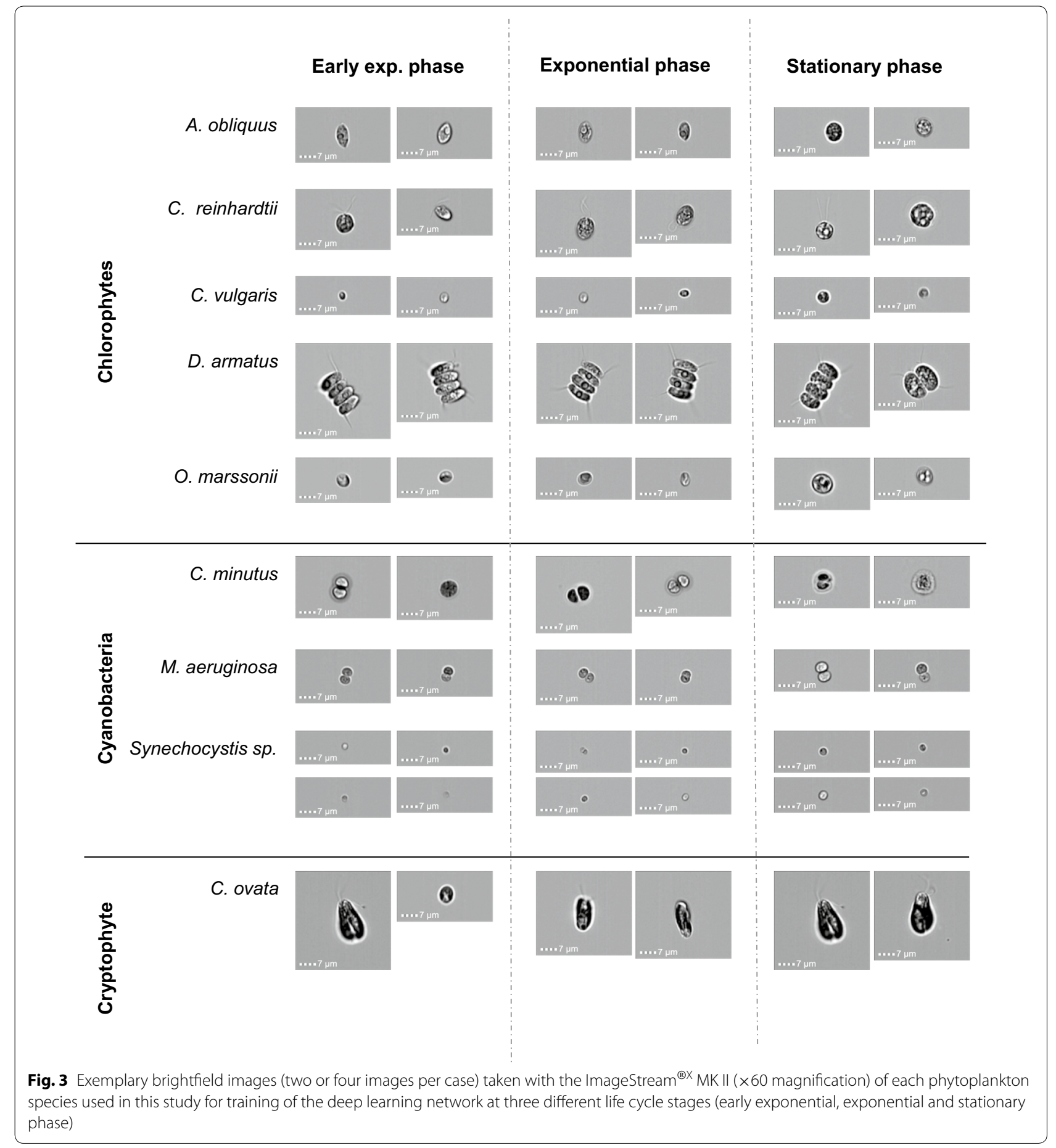

Validation of classifier performance via confusion matrices Confusion matrices were prepared to validate classifier performance, including potential species dependent misclassifications in more detail. In the confusion matrices performed, species are shown in rows versus the instances of the same species being predicted in columns. This allows a visualization of how a certain species was confused with others, if its accuracy was below $100 \%$ [43]. 


\section{Results}

Fluorescence in dependence of life cycle stage

The dataset used in this study, consisted of microscopic images for brightfield and Chl $a$ fluorescence, as well as interlinked standard flow cytometric data (fluorescence intensity values) taken with an imaging flow cytometer. To approximately simulate different pigmentation, like it is expected for laboratory cultured and field phytoplankton species, different life cycle stages were used. Figure 1 demonstrates the high variability of cellular fluorescence signals depending on life cycle stage of the batch culture. Besides C. vulgaris and O. marssonii, all species investigated show strong differences in a two-dimensional fluorescence plot especially between stationary and early exponential/exponential phase. C. ovata can even only be identified unambiguously during exponential phase, otherwise phycobilin absorption is so low, that when only fluorescence is used, misidentification with green algae is possible.

\section{Visual description of the species}

An impression of images used to train the $\mathrm{CNN}$ is provided in Fig. 3. Most investigated species show similar morphology (coccal: C. vulgaris, C. minutus, $C$. reinhardtii, $M$. aeruginosa, Synechocystis sp.; prolate spheroid/ellipsoidal: A. obliquus, O. marsonii, C. ovata; coenobia with prolate spheroid/ellipsoid cells: $D$. armatus) and belong to the taxonomic class of chlorophytes, cyanobacteria and cryptophytes. For each species, different life cycle stages were investigated. Most species at stationary phase have a different phenotypic appearance. Some species show a high accumulation of large intracellular granula of reserve material (e.g. starch) (A. obliquus, $C$. reinhardtii, O. marssonii), encystment (C. minutus) or an increase in cell size (M. aeruginosa, Synechocystis sp.). Based on morphological similarities, derived from brightfield images alone it was expected that confusion between $A$. obliquus, C. reinhardtii, C. vulgaris and $O$. marsonii, Synechocystis sp. as well as between C. minutus and $M$. aeruginosa could occur.

\section{Identification on species level}

The performance of the four different classifiers was evaluated in terms of accuracy and per-class-accuracy. Accuracy in Figs. 4 and 5 refers to the amount of all correctly classified

images in the test set, while averaged per-class accuracy indicates how well individual classes can be distinguished despite their imbalanced representation in our dataset. At the species level (Fig. 4), Chl $a$ fluorescence images alone show the lowest overall accuracy with $83 \%$ and per-class accuracy with $87 \%$, while combined images allow for the highest accuracy with $99 \%$ and a per-class accuracy of $98 \%$. Interestingly, even brightfield images alone result in accuracy and per-class accuracy of 97 and $93 \%$ respectively.

Species dependent misclassifications were visualized in more detail, in form of confusion matrices shown in Fig. 6. This figure illustrates the instances of an observed species in rows versus the instances of the same species

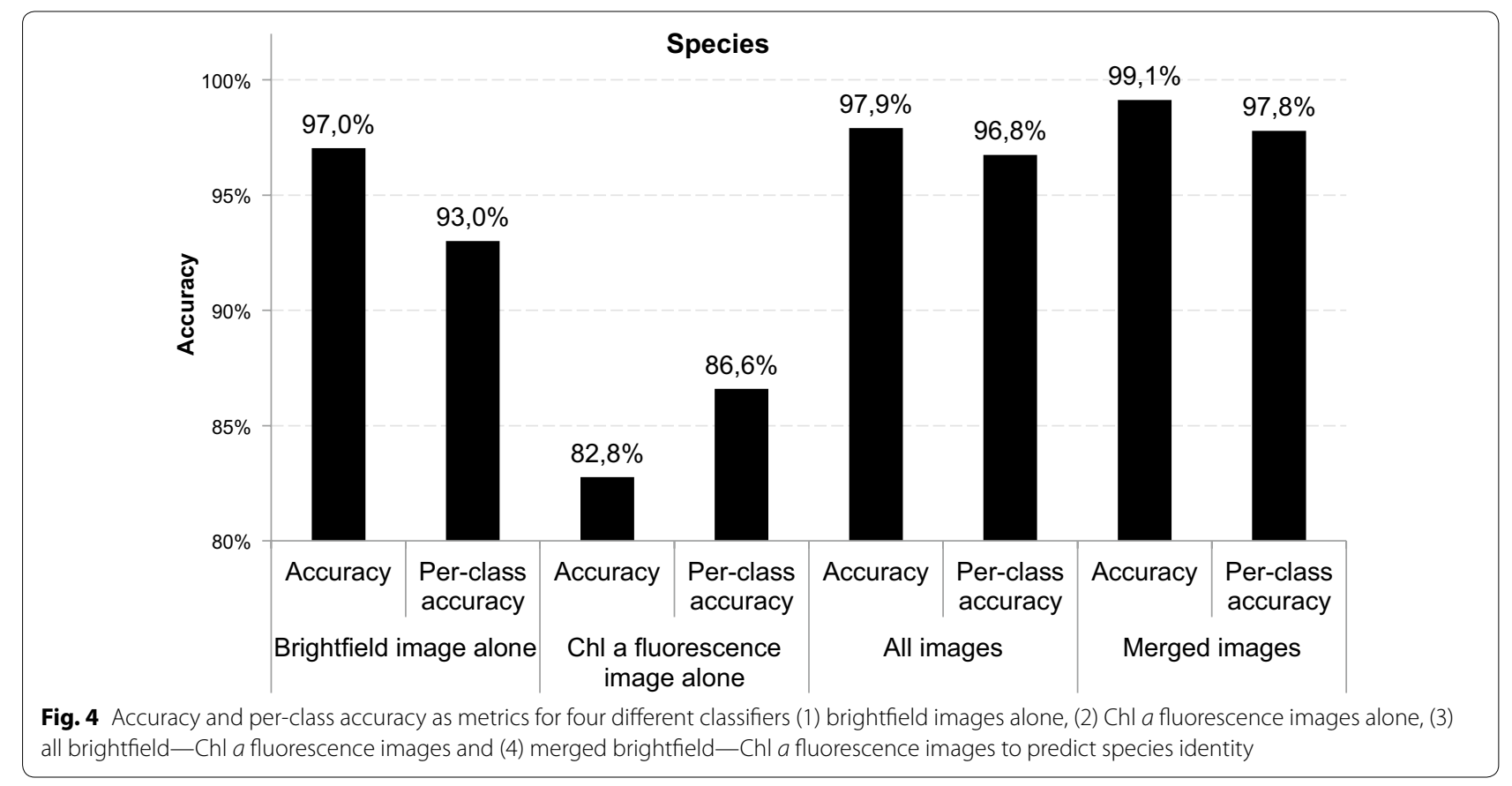




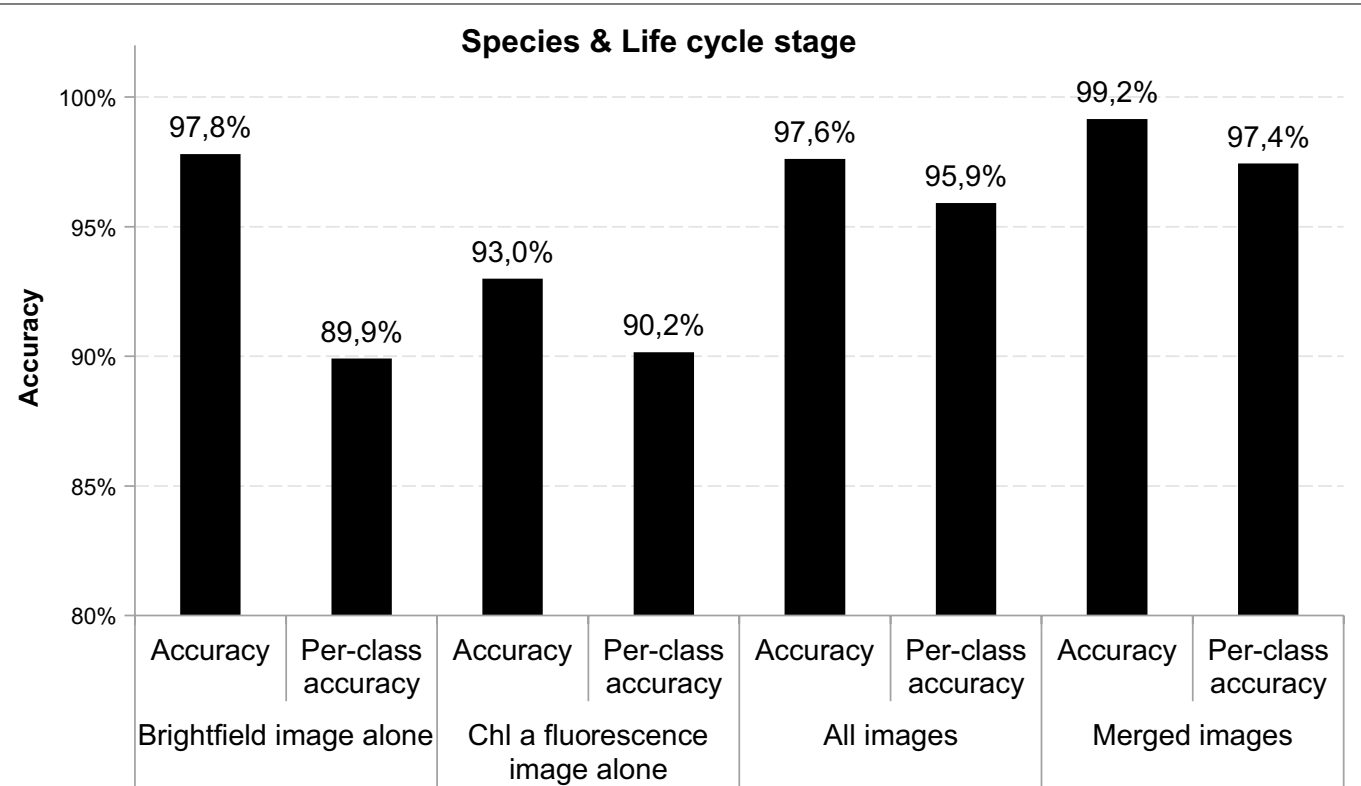

Fig. 5 Accuracy and per-class accuracy as metrics for four different classifiers (1) brightfield images alone, (2) Chl a fluorescence images alone, (3) all brightfield —Chl a fluorescence images and (4) merged brightfield—Chl $a$ fluorescence images to predict species identity and life cycle stage

being predicted in columns. We present one matrix per image type, visualizing how a certain species was confused with others, if its accuracy was below 100\% [43]. This is, for example, the case for C. ovata vs. O. marssonii for brightfield images. Using brightfield and Chl $a$ fluorescence images alone increases the tendency towards false classification, while the classifier with merged images decreases false classification. C. ovata achieved the lowest classification accuracy across all species (Fig. 6) and was often misclassified as O. marssonii for brigthfield images and C. vulgaris for Chl $a$ images. For the fourth category (merged images) C. ovata was confused with $A$. obliquus. Frequent confusion of $C$. ovata with green algae is on the one hand due to the fact that $C$. ovata is the least represented specie in the dataset (only 270 images could be provided for training) in contrast to all other species. On the other hand, Chl $a$ fluorescence of C. ovata is highly variable and shows high similarity in intensity to green algae when not growing in the exponential phase (Fig. 1).

\section{Identification on species level \& life cycle stage}

Classification of life cycle stage in combination with species identification shows a similar picture as species identification alone (Fig. 5). The classifier performance improved by including both, brightfield and Chl $a$ fluorescence images while $\mathrm{Chl} a$ fluorescence images alone yield the lowest accuracy. The classifier with highest accuracy and per-class accuracy is trained on merged brightfield and Chl $a$ images.
Misclassification for species and life cycle stage as visualized in Fig. 6 for all classifiers most frequently occurred in C. ovata vs. green algae. In general, most misclassifications were detected when brightfield or $\mathrm{Chl}$ $a$ fluorescence images were trained separately. Despite misclassification in C. ovata, Chl $a$ images alone led to misclassification of C. vulgaris and O. marssonii, as well as misclassification of different life cycle stages of $M$. aeruginosa. A combination of images, either by taking all images or merged images into account, improved classification in a way that high misclassification rates only occurred for C. ovata vs. C. vulgaris and O. marssonii.

\section{Discussion}

Phytoplankton species identification is a crucial part of water quality monitoring. But adequate monitoring is strongly limited by laborious microscopic techniques [17, 18].

Analytical flow cytometry can only partly be used to overcome the limitations of manual microscopy due to a large mismatch of taxonomic requirements and the highly compressed flow of cytometric single value outputs $[8,27]$. Shallow-learning artificial neural networks and other traditional classification techniques were nonetheless successfully applied to distinguish species based on their scattering properties and fluorescence emission signals $[1,5,6,19,50,51]$. Comparing accuracy of these approaches is complicated, because the size of datasets, the number of included parameters, the instruments and the image quality vary strongly among studies 


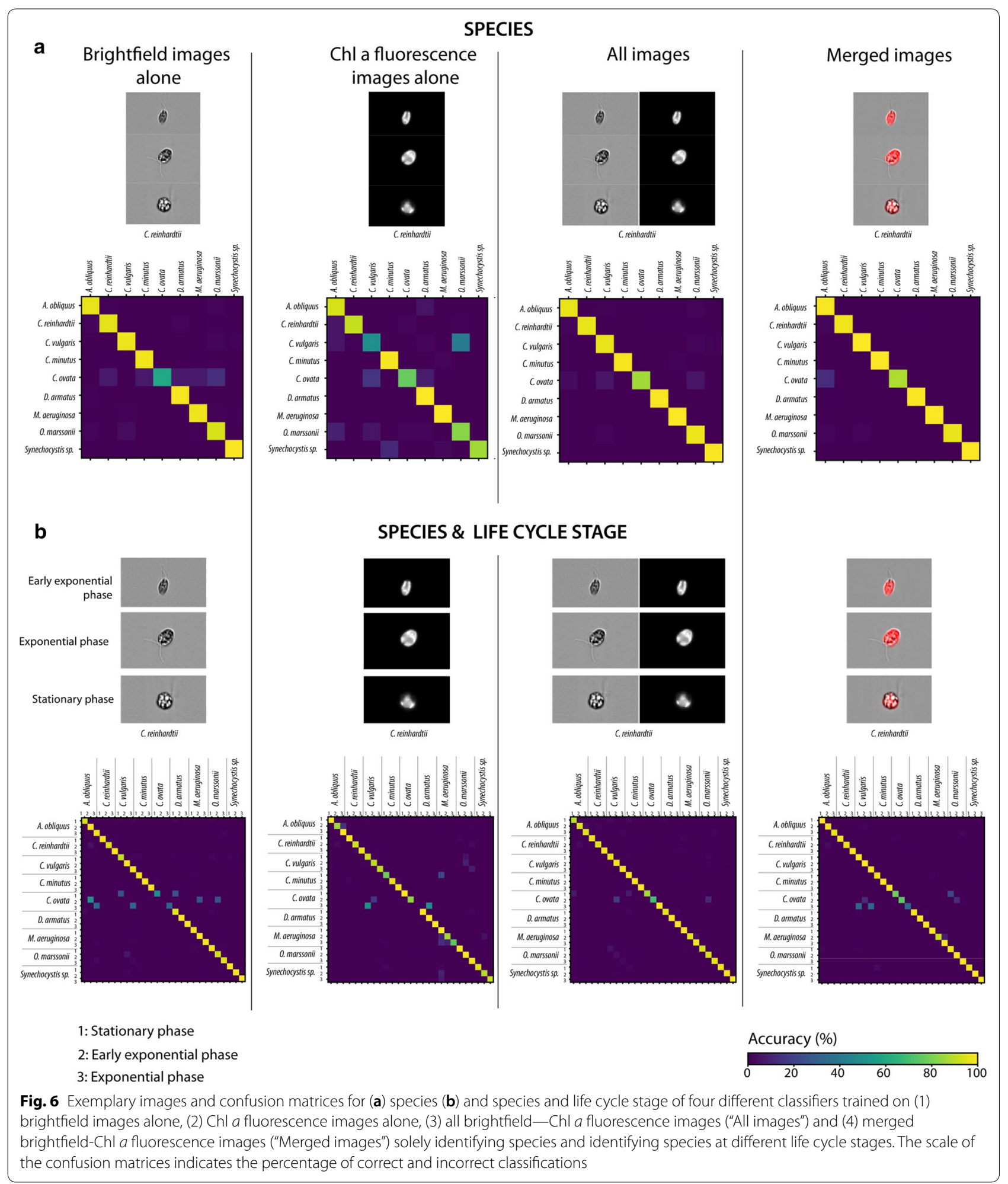

(cp. Table 2). Depending on the number of species and parameters considered, identification accuracy was between 70 and $98 \%$. Nevertheless, the utilized training datasets consisting of scatter and fluorescence signals of laboratory grown cultures (e.g. $[1,6]$ are not representative for natural field samples [50]). 


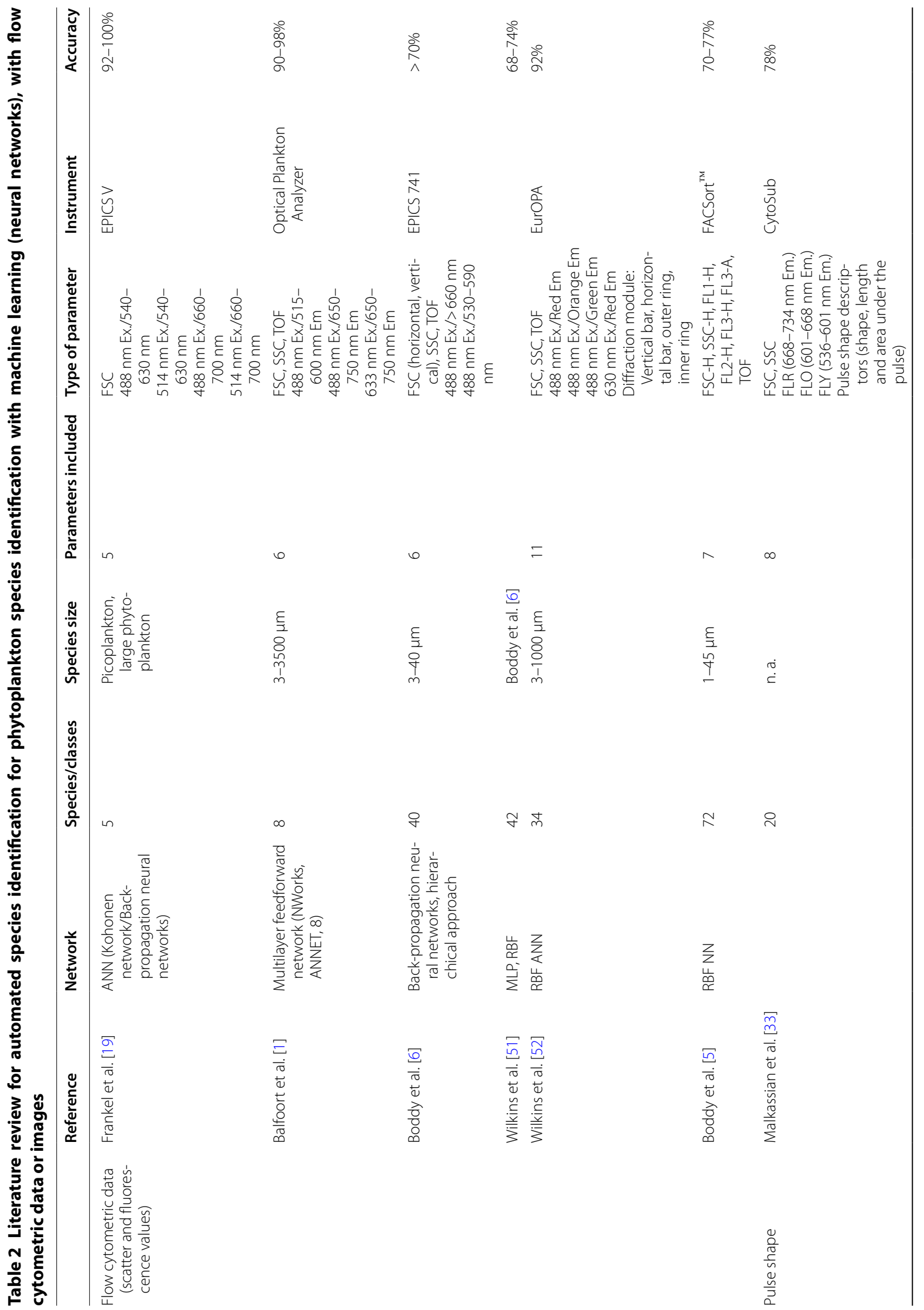




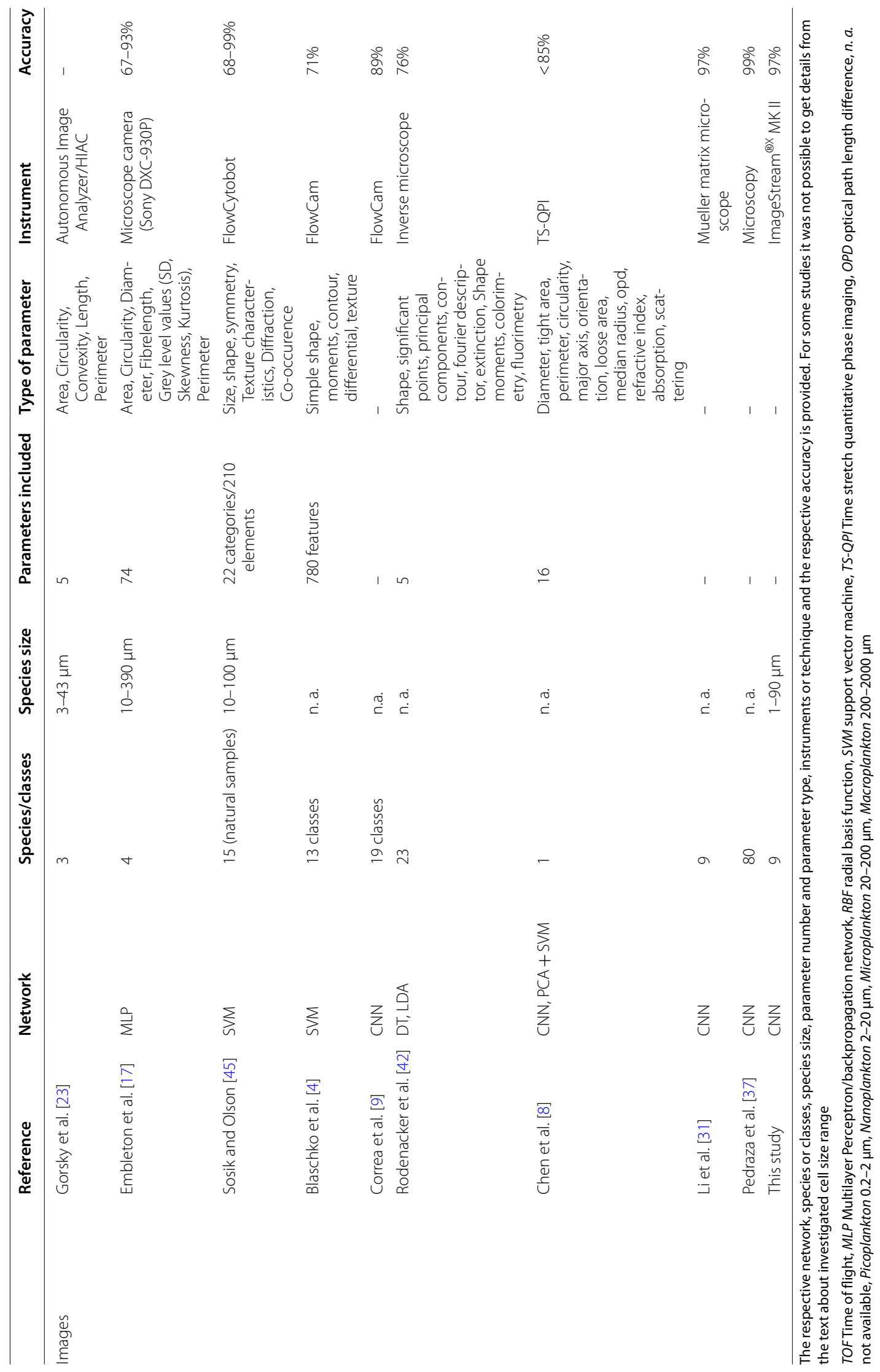


We argue that in contrast, image-based training datasets are more robust and suitable to reflect the large range of environmental conditions and mimick these conditions through different batch culture stages in this study. Gorsky et al. [23] pioneered research in this area using microscopic images and a set of simple geometric features to distinguish between three species (Prorocentrum micans, Nitzschia closterium and Hymenomonas elongata) of distinct size and shape. Blaschko et al. [4] achieved $50-70 \%$ classification accuracy with FlowCam images depicting twelve phytoplankton classes (centric diatoms, pennate diatoms, dinoflagellates, ciliates, unidentified cell, non-cell, Mesodinium, Laboea, Skeletonema, Thallasiosira, Thallasionema cf., Pseudonitzschia) and an "unknown" class using shape features, texture features, and contour. In the study by Blaschko et al. species were measured at a magnification of $4 \times$ or $10 \times$ and the cell size spectrum was between $\sim 10-60 \mu \mathrm{m}$. Rodenacker et al. [42] applied fluorescence imaging in their image acquisition system with a magnification of $20 \times$ and $40 \times$ to capture more information for discrimination between five classes of phytoplankton (Peridinium umbonatum, Cryptomonas erosa, Cryptomonas marsonii, Trachelomonas sp. and Ankistrodesmus sp.). The authors used species with a cell size in the range of 10-30 $\mu \mathrm{m}$ and report 58-93\% correct identifications. Sosik and Olson [45] used the FlowCytobot and a combination of image features including size, shape, symmetry, and texture characteristics to train a support vector machine (SVM) classifier (SVM classifier is a supervised learning model with associated learning algorithms) identifying 22 species (Asterionellopsis, Chaetoceros, Cylindrotheca, Cerataulina spp.+Dactyliosolen similar to Cerataulina spp. (DactFragCeratul), other Dactyliosolen, Dinobryon, Ditylum, Euglena, Guinardia, Licmophora, Phaeocystis, Pleurosigma, Pseudonitzschia, Rhizosolenia, Skeletonema, Thallasiosira, ciliate, detritus, dino, flagellate, other $<20 \mu \mathrm{m}$ and pennate) with an accuracy of $88 \%$. The study by Sosik and Olson used a species range between $\sim 5-400 \mu \mathrm{m}$, where most species were in a range between 20 and $50 \mu \mathrm{m}$. Schulze et al. [44] trained a traditional shallow-learning neural network classifier based on shape, texture and fluorescent features from microscopy phytoplankton images and reported a classification accuracy of $94.7 \%$ for ten taxa (Cyclotella menighiana, Anabaena sp., Chlorogonium elongatum, Cryptomonas ovata, Desmodesmus perforates, Staurastrum tetracerum, Botryococcus braunii, Pediastrum duplex, Trachelomonas volvocina, Crucigenia tetrapedia), a comparable cell size spectrum to this study. The studies by Blaschko et al. [4], Rodenacker et al. [42], Sosik and Olson [45] and Correa et al. [9] used species with a comparable large cell size, while this study should explore the lower edge of cell size range. We hypothesize that when small species can be well distinguished, larger species will be less problematic due to more detailed morphological structures.

In this study, we demonstrated a very high accuracy in species identification (99\% accuracy, per-class accuracy 97.8\%) with nine comparable small species by directly analyzing brightfield images (morphological information) and $\mathrm{Chl} a$ fluorescence images (chloroplast morphology and Chl $a$ fluorescence intensity) in combination rather than the highly compressed multi-variate scatter and fluorescence emission signals. Even when solely classifying based on brightfield images, the classifier delivered respectable accuracy (97\% accuracy, $93 \%$ per-class accuracy). A similar high accuracy was reached by Li et al. [31] (97\%) and Pedraza et al. [37] (99\%) by the use of microscopic images in combination with $\mathrm{CNN}$-training. Both authors did not use an imaging flow cytometer to collect the images, but used semi-automated microscopic systems. The high automation and in best case one-cell-at-once-analysis of the system used in this study is a major advantage for future phytoplankton analysis. Authors using other imaging flow cytometers (FlowCytobot, FlowCam) used SVM networks and reached accuracy of $68-99 \%$ [45] and 71\% [4]. The question is whether accuracy of image recognition achieved on images taken with the Flowcytobot or the FlowCAM could profit from CNNs. Here Correa et al. [9] could show $89 \%$ accuracy for FlowCAM images by using a CNN approach with 8 layers. An additional point for difficulties in comparison with the current study is the point that Sosik and Olson [45], Blaschko et al. [4] and Correa et al. [9] used phytoplankton images from field measurements with more artifacts and trash. In future studies the applicability of the system used in this study shall be investigated for field measurements. The $60 \times$ magnification was demonstrated as suitable magnification to collect images of cells in the size range of 1-90 $\mu \mathrm{m}$, representing a crucial part of natural phytoplankton communities [45]. For larger genera (data not shown), e.g. Asterionella, Anabaena or Planktothrix the $20 \times$ magnification would be more useful due to a larger field of view.

It would in theory be possible to include additional fluorescence images from different excitation and emission channels, but the accuracy could be only marginally improved, because it was already really high. However, additional image channels may further increase the robustness of species identification and especially for future issues dealing with higher complexity in natural samples it could be relevant to include images from additional fluorescence channels. This needs to be evaluated with a larger dataset, containing more taxonomic groups and species. The extra channels are nevertheless relevant for taxonomic pre-sorting of data by assigning 
larger taxonomic groups and to subsequently apply a deep learning approach identifying species for these specific taxonomic groups. A balanced number of images (the same number of images for each species) for training would be ideal, to better compare classifier accuracy. Alternatively, per-class accuracy was established as comparative parameter, because all images collected should be used for training.

In this study, we took as additional prediction parameter 'life cycle stage' into account. Batch cultivation, starting with a small inoculum, a lag, early exponential, exponential and stationary phase is a good way to mimic different life cycle stages, which could also occur under field conditions. It is expected that the physiological performance varies under these different cultivation phases, whereby it is highest during exponential phase and lowest during stationary phase. Especially the stationary phase is a phase of high nutrient and light deficiency. Like described in other studies as well, cells are able to prepare for long phases of deficiency by accumulating storage compounds, like starch grains or cyanophycin [15, $16,40]$. In our study, we found starch grain like granules in stationary phase cells of $A$. obliquus, C. reinhardtii, $D$. armatus, and $O$. marssonii. An additional indication of cellular adaption to nutrient deficiency is a large cell size [15], like observed for all species with an accumulation of starch grains and C. vulgaris, M. aeruginosa and Synechocystis sp. Long et al. [32] suggest cell size increase as a good stress indicator. For $C$. minutus a thick wall layer could be identified from the images as kind of encystment, being an adaption to long term persistence, like described by Ellegaard and Ribeiro [16].

In addition to species identification alone, estimation of life cycle stage, nutrient deficiency or much more general a stressor could be a valuable feature for biotechnological applications or field studies by informing about ongoing primary production or grazing effects. Furthermore, evidence for a mixing event or the end of the vegetation period could be concluded if encysted, enlarged or storage material containing cells are detected in the water body. In biotechnological approaches life cycle stage monitoring could be valuable for process control.

Deep learning artificial neural networks automate the otherwise subjective critical feature extraction step by learning a suitable representation of the training data and by systematically developing a robust classification model. In contrast to existing approaches there is a higher similarity to taxonomic approaches by considering detailed morphological information as well as chloroplast morphology, color and intensity. Deep learning approaches show remarkable performance, but the process behind is kind of a black box and transformation into white-box is in the early stages [34]. It is important to be aware of artifacts, like probably occurring in the background of the phytoplankton images, taken with the imaging flow cytometer. To consider and exclude potential influence of background on species identification, we carefully augmented images during training, in terms of brightness, saturation, contrast and hue-values, to induce variations of the image background. In comparison to other image recognition tasks, e.g. with images from natural landscapes, the background in images from an imaging flow cytometer used is much more homogeneous and stable, because optical settings are calibrated each day before the start of the measurements.

The case of $C$. ovata shows that 20-63 images per class were not sufficient to train the network sufficiently. In contrast, $D$. armatus in early exponential phase had only 235 images available, but is properly classified. Therefore it is suggested to collect at least 200 images in order to develop a robust species recognition classifier. However, further evaluation in this direction is required since the minimum number of training images may also be species-dependent, for example species with special characteristics may require less images. If sufficient images are available, it can be expected that the learning curve of machine learning approaches is more predictable [25] than human learning curve and can be done in shorter time, due to continuous training. By using one classifier for species identification, much higher objectivity is given. However, even though there is doubt about correct identification, automatic image recording allows archiving data and to perform a subsequent proof of identification at a later point in time [42].

The approach suggested in this study should be extended to a larger number of species, but could then be helpful for many operators, responsible for phytoplankton monitoring, e.g. within the European Water Framework Directive, Great Lakes Phytoplankton Monitoring by the Environmental Protection Agency of the United States (EPA) or the monitoring program of ballast water of the International Maritime Organization. The method enables the operator to get an automated instantaneous, archivable, objective and quantitative information about water quality relevant phytoplankton communities.

\section{Conclusion}

There are reasonable doubts concerning the reliability of AFC for indicator-taxa identification due to low taxonomic resolution, but these concerns may be overcome given there is a microscopic image for each single measured cell.

In this study, we presented for the first time an automated approach for identifying species and their life cycle stage utilizing state of the art machine learning techniques (CNNs), working best when using a 
classifier trained on merged brightfield and Chl $a$ fluorescence images. Species identity and in addition life cycle stage have been predicted successfully, while flow cytometric measurements provide quantitative data for each single species.

Our study is an initial milestone for future improvement, e.g. with a larger set of species. The configuration of the image-based cytometer used, would also allow to cover a broader phytoplankton size spectrum (cells up to $100 \mu \mathrm{m}$ width, when arranged along the fluid stream in longest axial dimension) by taking images at lower magnifications $(20 \times$ or $40 \times)$. In addition, the robustness of classifications could be further improved by pooling different measurements taken at different times and environmental conditions, as well adding additional fluorescence images. In this study, we demonstrated that a CNN classifier can recognize species, even if their $\mathrm{Chl} a$ fluorescence pattern was extremely different, depending on the respective life-cycle stage.

Manifold necessary monitoring tasks, like ballast water monitoring and other national and international monitoring programs, could potentially profit from the suggested combination of high through-put imaging flow cytometry and deep learning. In future, a detailed evaluation of the method against traditional microscopic species identification is needed.

\begin{abstract}
Abbreviations
AFC: analytical flow cytometry; ANN: artificial neural networks; Chl a: chlorophyll $a$; CNN: convolutional neural networks; FCM: flow cytometry; FSC: Forward scatter signal; IFC: imaging flow cytometry; SSC: Sideward scatter signal; SVM: support vector machine.
\end{abstract}

\section{Authors' contributions}

SD is main author of the study and performed all phytoplankton experimental work including flow cytometry and drafted the manuscript. SD and JW initiated the study. DB performed data preparation and processing, classifier training and evaluation. JW helped to interpret the data and substantially contributed to the manuscript. PM designed, instructed and approved the deep learning workflow. All authors read and approved the final manuscript.

\section{Author details \\ ${ }^{1}$ Department of Physiological Diversity, Helmholtz-Centre for Environmental Research-UFZ, Permoserstraße 15, 04318 Leipzig, Germany. ${ }^{2}$ Department of Physiological Diversity, German Centre for Integrative Biodiversity Research- iDiv, Deutscher Platz 5a, 04103 Leipzig, Germany. ${ }^{3}$ Software Engineering for Safety-Critical Systems Group, Technische Universität IImenau, Ehrenberg- straße 29, 98693 IImenau, Germany. ${ }^{4}$ Department of Biochemical Integration, Max-Planck-Institute for Biogeochemistry, Hans-Knöll-Straße 10, 07745 Jena, Germany.}

\section{Acknowledgements}

JW, DB and PM are funded by the German Ministry of Education and Research (BMBF) Grants: 01LC1319A and 01LC1319B.

\section{Competing interests}

The authors declare that they have no competing interests.

\section{Availability of data and materials}

The datasets used and/or analyzed during the current study are available from the corresponding author on reasonable request. The data presented in the publication will be publicly available in case of manuscript acceptance.

\section{Consent to publish}

Not applicable.

\section{Ethics approval and consent to participate}

Not applicable.

\section{Funding}

JW, DB and PM are funded by the German Ministry of Education and Research (BMBF) Grants: 01LC1319A and 01LC1319B.

\section{Publisher's Note}

Springer Nature remains neutral with regard to jurisdictional claims in published maps and institutional affiliations.

Received: 20 March 2018 Accepted: 22 November 2018

Published online: 03 December 2018

\section{References}

1. Balfoort H, Snoek J, Smiths J, Breedveld L, Hofstraat J, Ringelberg J. Automatic identification of algae: neural network analysis of flow cytometric data. J Plankton Res. 1992;14(4):575-89.

2. Basiji DA, Ortyn WE, Liang L, Venkatachalam V, Morrissey P. Cellular image analysis and imaging by flow cytometry. Clin Lab Med. 2007;27(3):653-70.

3. Bischoff HW. Some soil algae from enchanted rock and related algal species. Phycological. 1963;studies:95.

4. Blaschko MB, Holness G, Mattar MA, Lisin D, Utgoff PE, Hanson AR, et al. Automatic in situ identification of plankton. In: Seventh IEEE Workshops on application of computer vision, 2005. WACV/MOTIONS'05 1, volume 1; 2005. p. 79-86.

5. Boddy L, Morris C, Wilkins M, Al-Haddad L, Tarran G, Jonker R, et al. Identification of 72 phytoplankton species by radial basis function neural network analysis of flow cytometric data. Marine Ecol Prog Ser. 2000;195:47-59.

6. Boddy L, Morris C, Wilkins M, Tarran G, Burkill P. Neural network analysis of flow cytometric data for 40 marine phytoplankton species. Cytometry Part A. 1994;15(4):283-93.

7. Boddy L, Wilkins MF, Morris CW. Pattern recognition in flow cytometry. Cytometry Part A. 2001;44(3):195-209.

8. Chen CL, Mahjoubfar A, Tai L-C, Blaby IK, Huang A, Niazi KR, et al. Deep learning in label-free cell classification. Sci Rep. 2016;6:21471.

9. Correa I, Drews P, Botelho $S$, de Souza MS. Deep learning for microalgae classification. In: Machine learning and applications (ICMLA), 2017 16th IEEE international conference on machine learning and applications; 2017. p. 20-5

10. Cullen JJ, Lewis MR. The kinetics of algal photoadaptation in the context of vertical mixing. J Plankton Res. 1988;10(5):1039-63.

11. Culverhouse PF. Human and machine factors in algae monitoring performance. Ecol Inf. 2007:2(4):361-6.

12. Culverhouse PF, Williams R, Benfield M, Flood PR, Sell AF, Mazzocchi MG, et al. Automatic image analysis of plankton: future perspectives. Mar Ecol Prog Ser. 2006;312:297-309.

13. Culverhouse PF, Williams R, Reguera B, Herry V, González-Gil S. Do experts make mistakes? A comparison of human and machine identification of dinoflagellates. Mar Ecol Prog Ser. 2003;247:17-25.

14. Dashkova V, Malashenkov D, Poulton N, Vorobjev I, Barteneva NS. Imaging flow cytometry for phytoplankton analysis. Methods. 2017;1 12:188-200.

15. van Donk E, Lürling $M$, Hessen D, Lokhorst G. Altered cell wall morphology in nutrient-deficient phytoplankton and its impact on grazers. Limnol Oceanogr. 1997;42(2):357-64.

16. Ellegaard M, Ribeiro $S$. The long-term persistence of phytoplankton resting stages in aquatic 'seed banks'. Biol Rev. 2017;93(1):166-83. 
17. Embleton K, Gibson C, Heaney S. Automated counting of phytoplankton by pattern recognition: a comparison with a manual counting method. J Plankton Res. 2003;25(6):669-81.

18. First MR, Drake LA. Performance of the human "counting machine": evaluation of manual microscopy for enumerating plankton. J Plankton Res. 2012;34(12):1028-41.

19. Frankel DS, Olson RJ, Frankel SL, Chisholm SW. Use of a neural net computer system for analysis of flow cytometric data of phytoplankton populations. Cytometry Part A. 1989;10(5):540-50.

20. Fujiki T, Taguchi S. Variability in chlorophyll a specific absorption coefficient in marine phytoplankton as a function of cell size and irradiance. J Plankton Res. 2002;24(9):859-74.

21. Geider RJ. Quantitative phytoplankton physiology: implications for primary production and phytoplankton growth. ICES Mar Sci Symp. 1993;197:52-62.

22. Geider R, Maclntyre H, Kana T. Dynamic model of phytoplankton growth and acclimation: responses of the balanced growth rate and the chlorophyll a: carbon ratio to light, nutrient-limitation and temperature. Marine Ecol Prog Ser. 1997;148:187-200.

23. Gorsky G, Guilbert P, Valenta E. The autonomous image analyzer-enumeration, measurement and identification of marine phytoplankton. Marine Ecol Prog Ser. 1989;58:133-42.

24. He K, Zhang X, Ren S, Sun J. Identity mappings in deep residual networks. In: European conference on computer vision; 2016. p. 630-45.

25. Hestness J, Narang S, Ardalani N, Diamos G, Jun H, Kianinejad H, et al. Deep learning scaling is predictable, empirically; 2017. arXiv preprint. arXiv:1712.00409.

26. Hildebrand M, Davis A, Abbriano R, Pugsley HR, Traller JC, Smith SR, et al. Applications of imaging flow cytometry for microalgae. In: Barteneva NS, Vorobjev IA, editors. Imaging flow cytometry. New York: Humana Press; 2016. p. 47-67.

27. Hillebrand HD-D. Biovolume calculation for pelagic and benthic microalgae. J Phycol. 1999;35:403-24.

28. Hofstraat J, Van Zeijl W, De Vreeze M, Peeters J, Peperzak L, Colijn F, et al. Phytoplankton monitoring by flow cytometry. J Plankton Res. 1994;16(9):1197-224.

29. John DM, Whitton BA, Brook AJ. The freshwater algal flora of the British Isles: an identification guide to freshwater and terrestrial algae, vol. 1. Cambridge: Cambridge University Press; 2002.

30. Karlson B, Gohde A, Cusack C, Bresnan E. Introduction to methods for quantitative phytoplankton analysis. In: Karlson BC, Cusack C, Bresnan E, editors. Microscopic and molecular methods for quantitative phytoplankton analysis. Paris: UNESCO; 2010.

31. Li X, Liao R, Zhou J, Leung PT, Yan M, Ma H. Classification of morphologically similar algae and cyanobacteria using Mueller matrix imaging and convolutional neural networks. Appl Opt. 2017:56(23):6520-30.

32. Long BM, Jones GJ, Orr PT. Cellular microcystin content in N-limited Microcystis aeruginosa can be predicted from growth rate. Appl Environ Microbiol. 2001;67(1):278-83.

33. Malkassian A, Nerini D, van Dijk MA, Thyssen M, Mante C, Gregori G. Functional analysis and classification of phytoplankton based on data from an automated flow cytometer. Cytometry part A. 2011;79(4):263-75.

34. Min S, Lee B, Yoon S. Deep learning in bioinformatics. Brief Bioinform. 2017;18(5):851-69.

35. Padisak J, Borics G, Grigorszky I, Soroczki-Pinter E. Use of phytoplankton assemblages for monitoring ecological status of lakes within the water framework directive: the assemblage index. Hydrobiologia. 2006;553(1):1-14.

36. Palmer CM. A composite rating of algae tolerating organic pollution 2. J Phycol. 1969;5(1):78-82.

37. Pedraza A, Bueno G, Deniz O, Cristóbal G, Blanco S, Borrego-Ramos M. Automated diatom classification (Part B): a deep learning approach. Appl Sci. 2017;7(5):460.
38. Phillips G, Lyche-Solheim A, Skjelbred B, Mischke U, Drakare S, Free G, et al. A phytoplankton trophic index to assess the status of lakes for the water framework directive. Hydrobiologia. 2013;704(1):75-95.

39. Pomati F, Jokela J, Simona M, Veronesi M, Ibelings BW. An automated platform for phytoplankton ecology and aquatic ecosystem monitoring. Environ Sci Technol. 2011;45(22):9658-65.

40. Pulz O, Gross W. Valuable products from biotechnology of microalgae. Appl Microbiol Biotechnol. 2004;65(6):635-48.

41. Reynolds C. What factors influence the species composition of phytoplankton in lakes of different trophic status? Hydrobiologia. 1998;369:11-26.

42. Rodenacker K, Hense B, Jütting U, Gais P. Automatic analysis of aqueous specimens for phytoplankton structure recognition and population estimation. Microsc Res Tech. 2006;69(9):708-20.

43. Rzanny M, Seeland M, Wäldchen J, Mäder P. Acquiring and preprocessing leaf images for automated plant identification: understanding the tradeoff between effort and information gain. Plants Methods. 2017;13:97.

44. Schulze K, Tillich UM, Dandekar T, Frohme M. PlanktoVision-an automated analysis system for the identification of phytoplankton. BMC Bioinform. 2013;14(1):115

45. Sosik HM, Olson RJ. Automated taxonomic classification of phytoplankton sampled with imaging-in-flow cytometry. Limnol Oceanogr Methods. 2007;5(6):204-16.

46. Staub R. Ernährungsphysiologisch-autökologische Untersuchungen an der planktischen Blaualge Oscillatoria rubescens DC. Aquat Sci Res Across Bound. 1961;23(1):82-198.

47. Suthers IM, Rissik D. Plankton: A guide to their ecology and monitoring for water quality. Clayton: CSIRO Publishing; 2009.

48. Swanstrom JA, Bruckman LS, Pearl MR, Abernathy E, Richardson TL, Shaw $\mathrm{TJ}$, et al. Taxonomic classification of phytoplankton with multivariate optical computing, part II: design and experimental protocol of a shipboard fluorescence imaging photometer. Appl Spectrosc. 2013;67(6):630-9.

49. Wäldchen J, Rzanny M, Seeland M, Mäder P. Automated plant species identification-Trends and future directions. PLoS Comput Biol. 2018;14(4):e1005993.

50. Wilkins MF, Hardy SA, Boddy L, Morris CW. Comparison of five clustering algorithms to classify phytoplankton from flow cytometry data. Cytometry Part A. 2001:44(3):210-7.

51. Wilkins MF, Morris C, Boddy L. A comparison of radial basis function and backpropagation neural networks for identification of marine phytoplankton from multivariate flow cytometry data. Bioinformatics. 1994:10(3):285-94.

52. Wilkins M, Boddy L, Morris C, Jonker R. Identification of phytoplankton from flow cytometry data by using radial basis function neural networks. Appl Environ Microbiol. 1999;65(10):4404-10.

53. Willen E. Phytoplankton in water quality assessment-an indicator concept. Hydrol limnol Aspects Lake Monit. 2000;57:80.

54. Yosinski J, Clune J, Bengio Y, Lipson H. How transferable are features in deep neural networks? In: Proceedings of the 27th international conference on neural information processing systems, vol. 2. Cambridge: MIT Press; 2014. p. 3320-8.

Ready to submit your research? Choose BMC and benefit from:

- fast, convenient online submission

- thorough peer review by experienced researchers in your field

- rapid publication on acceptance

- support for research data, including large and complex data types

- gold Open Access which fosters wider collaboration and increased citations

- maximum visibility for your research: over $100 \mathrm{M}$ website views per year

At BMC, research is always in progress.

Learn more biomedcentral.com/submissions 